\title{
Difficultés Relatives A L'enseignement-Apprentissage De La Géologie En Classes Secondaires Qualifiantes Cas De La Délégation d'Inzegane Ait Melloul
}

\author{
Khadija Kaid Rassou, \\ Fouad Khiri, \\ Mohamed Benbrahim,
} Enseignants Chercheurs et Formateurs des Futurs, Enseignants des Sciences de la Vie et de la Terre (SVT).

Equipe de Géologie et Didactique de Géologie

Centre Régional des Métiers de l'Education et de la Formation (CRMEF), Sous Massa, Avenue My Abdallah B.P 106, Inezgane, Maroc

\section{Younes Tamraoui, Hafida Elberrani, Maryem Anfour,}

Enseignants des Sciences de la Vie et de la Terre, Cycle de L'enseignement Secondaire Qualifiant. Ministère de l'Education Nationale, de la Formation Professionnelle, de l'Enseignement Supérieur et de la Recherche Scientifique, Maroc

doi: 10.19044/esj.2017.v13n18p294 URL:http://dx.doi.org/10.19044/esj.2017.v13n18p294

\section{Abstract}

The present study attempts to identify the difficulties of teaching and learning geology in Moroccan secondary qualifying classes. Teachers of the Life Sciences and Earth Sciences and their pupils at the Inzegane Ait Melloul delegation were administered questionnaires. Based on the analysis of the themes taught and the typologies of obstacles evoked, a bibliographic synthesis allowed us to list the notions which are likely to pose the difficulties presumed in geology. Thus, 23 open and closed questions were distributed to seven teachers. Also, ten questions were distributed to students. The results showed that both pupils and teachers experience several difficulties in the teaching and learning of geology. Thus, the various means of continuing education in geology which are made available to teachers were disqualified. It involves only a minority of them. This is in addition to the complexity of the geological content and the insufficiency of its hourly volume. The excessive number of pupils causes the classes to be overloaded, 
in which the weak prerequisite is added in the geology of these pupils. The difficulties of learning geology are largely related to the relationship that this discipline maintains with space and long time. This, therefore, make it difficult for pupils to understand several complex geological phenomena. The ineffectiveness of teaching methods tailored to the classroom by teachers, the absence, failure or lack of mobilization of teaching and ICT resources, inadequacy or absence of field trips, and the manipulations and negligence of scientific modeling in majority of the classes surveyed influences the learning of geology as well as the students' interest in these courses. This makes it a boring and unwelcome matter for most students.

Keywords: Teaching, learning, Earth Sciences, difficulties, qualifying secondary classes

\section{Résumé}

La présente étude tente de déceler les difficultés de l'enseignement et de l'apprentissage de la géologie en classe secondaires qualifiantes marocaines. Des enseignants de la matière des Sciences de la Vie et de la Terre et ses élèves à la délégation Inzegane Ait Melloul sont soumis à des questionnaires; réalisés à partir de l'analyse des thèmes enseignés et des typologies d'obstacles évoquées suite à une synthèse bibliographique, ce qui nous a permis de lister les notions susceptibles de poser les difficultés pressenties en géologie. Ainsi 23 questions tant ouvertes que fermées, réparties sur sept principales parties ont été proposées aux enseignants et 10 questions réparties sur cinq parties ont été présentées aux élèves. Les résultats ont montré que l'enseignement et l'apprentissage de la géologie sont confrontés à plusieurs difficultés aussi bien pour les élèves que pour les enseignants. Ainsi les moyens de la formation continue en géologie qui sont mis à la disposition des enseignants sont déqualifiés et ne concernent qu'une minorité d'entre eux, en plus de la complexité du contenu géologique et l'insuffisance de son volume horaire, l'effectif pléthorique des élèves provoquant la surcharge des classes à quoi s'ajoute le faible prérequis en géologie de ces élèves. Les difficultés de l'apprentissage de la géologie sont largement liées aux rapports que cette discipline entretient avec l'espace et le temps long, et qui font que les élèves trouvent des difficultés à appréhender plusieurs phénomènes géologiques complexes. Ainsi l'inefficacité des méthodes d'enseignement adaptées en classe par les professeurs, l'absence, défaillance ou non mobilisation des ressources pédagogiques et des TICE, l'insuffisance ou l'absence des sorties de terrain, des manipulations et la négligence de la modélisation scientifique dans la majorité des classes interrogées, influencent les apprentissages de la géologie ce qui influe 
l'intérêt porté par les élèves vis-à-vis de ses cours ce qui la rend, une matière ennuyeuse et mal aimée par la majorité des élèves.

Mots-clés: Enseignement, apprentissage, géologie, difficultés, Classes secondaires

\section{Introduction}

La Géologie est une science qui vise à dégager les concepts clefs permettant d'expliquer le fonctionnement de notre planète. Elle comporte plusieurs disciplines à savoir la pétrologie, la géophysique, la géochimie, la paléontologie, la stratigraphie, l'hydrogéologie.

L'enseignement de la géologie au secondaire qualifiant est une phase de spécialisation qui donne à l'élève les notions scientifiques et techniques nécessaires lui permettant d'accéder à l'enseignement supérieur. Il fait appel à l'expérimentation contrôlée fondée principalement sur le travail au laboratoire, ainsi qu'à l'observation de terrain et à la modélisation. Actuellement, la géologie occupe une place plus au moins importante au sein des programmes des Sciences de la Vie et de la Terre enseignés dans ce cycle.

L'enseignement de la géologie dont la thématique s'articule autour de la dynamique terrestre, a fait l'objet d'une enquête auprès des enseignants et élèves du secondaire qualifiant, délégation Inzegane Ait Melloul, afin d'approcher de près l'acte de l'enseignement afin de souligner les différentes difficultés rencontrées lors de l'enseignement et l'apprentissage de cette discipline.

\section{Problématique et Objectifs}

L'enseignement de la géologie au Maroc n'a pas évolué et présente plusieurs difficultés (Lakhloufi, 2014). D'après les pratiques de classe, la majorité des professeurs de la matière des Sciences de la Vie et de la Terre (SVT), que nous rencontrons dans les établissements scolaires lors des stages de Mise en Situations Professionnelles (MSP) de nos professeurs stagiaires, constatent que les élèves sont très peu impliqués dans les cours de la géologie alors qu'ils s'intéressent mieux au cours de la biologie. De plus, l'enseignement de la géologie semble problématique aussi pour les enseignants qui doivent enseigner la géologie sans avoir été eux-mêmes parfois formés à l'enseignement de cette discipline. De ce fait l'enseignement et la compréhension de la géologie semblent confrontés à plusieurs difficultés aussi bien pour les élèves que pour les enseignants.

C'est donc pour toutes ces raisons et bien d'autres qu'il nous semble important de chercher les origines des difficultés de l'enseignement et de 
l'apprentissage de la géologie au Maroc en prenant le cas la délégation d'Inzegane Ait Melloul.

Ce travail s'inscrit ainsi dans une perspective visant les objectifs suivants :

- Identifier les difficultés et obstacles de l'enseignement et de l'apprentissage de la géologie rencontrés par les enseignants et par leurs élèves en classes secondaires qualifiantes marocaines;

- Décrire les pratiques de classe afin d'évaluer les besoins des enseignants en termes de ressources et de formation en fonction de ces difficultés.

- Effectuer des préconisations aux concepteurs des programmes SVT au Maroc afin d'améliorer sa qualité et son efficacité.

\section{Cadre Théorique}

\section{Synthèse des Travaux Antérieurs}

Une synthèse bibliographique des études qui ont été menées un peu partout dans le monde a été réalisée. L'appréhension du temps et de l'espace, la modélisation des phénomènes, sont des points retenus comme principales difficultés didactiques par les auteurs qui se sont penchés sur l'enseignement de la géologie. Ainsi d'après Gould (1990), ces difficultés sont largement liées aux rapports que la discipline entretient avec le temps : difficultés à appréhender des temps longs, à élaborer un raisonnement diachronique, à saisir le rôle de la contingence dans l'histoire géologique. Selon Gohau (1990), « une des difficultés de l'enseignement de la géologie tient à notre incapacité à prendre conscience des immenses durées du passé de la Terre [...] Nous sommes aussi démunis devant ces chronologies énormes que devant l'immensité de l'espace en astronomie ». Monchamp et SauvageotSkibine (1995) relèvent que le fixisme constitue un obstacle à la compréhension de phénomènes dynamiques paraissant statiques à l'échelle humaine.

Les travaux de C. Orange (1999) soulignent les difficultés qu'ont les élèves à articuler les références empiriques et énoncés théoriques lors de travaux sur le terrain. Ces travaux en 2003, mettent l'accent sur le rôle de l'investigation au laboratoire et sur l'observation de terrain.

Trend (2000) souligna la difficulté à appréhender les échelles de temps impliquées. Les études de ont montré que les élèves avaient des difficultés à appréhender les temps longs et la vitesse de réalisation des phénomènes géologiques (Sanchez \& Prieur, 2006; Raab \& Frodeman, 2002).

D'autres travaux, tendent à montrer que le statut du modèle scientifique pose problème aux enseignants de sciences de la vie et de la Terre de 1'enseignement secondaire qualifiant (Sanchez \& Devallois, 2004; 
Sanchez, 2008) considère les modèles comme des instruments de connaissance permettant aux élèves, dans le cadre de la résolution d'un problème scientifique, de conduire leurs investigations et de donner du sens aux tâches dans lesquelles ils s'engagent.

Les résultats d'une étude effectuée en 2004 par l'équipe de recherche technologique en éducation à l'INRP de Lyon en France, ont montré que le manque des travaux de terrains et des pratiques expérimentales en classe ainsi que l'absence des formations initiales ou continues des professeurs des Sciences de la Vie et de la Terre en géologie sont les principales difficultés qui influencent les apprentissages géologiques. Dans le même cadre une enquête qui a été conduite par a montré que les enseignants qui organisent une classe de terrain ont des difficultés à organiser un travail d'investigation scientifique (Sanchez \& Fontanieu, 2005).

Ainsi ont montré que la sortie de terrain est absolument nécessaire afin de lutter contre les obstacles liés aux échelles (de taille et de temps) en sciences de la Terre (Lamarti et al., 2009).

Au Liban, une étude menée par a mis en évidence la présence de plusieurs obstacles épistémologiques, didactiques, idéologiques, économiques, curriculaires et professionnelles pouvant être à la base des résistances que rencontre l'enseignement de cette discipline (Chalak \& El Hage, 2011).

Une étude menée en Arabie Saoudite par a montré que l'absence des laboratoires géologiques au sein des établissements scolaires et l'absence des classes de terrain et des sorties des centres spécialisé en géologie constituent les principales causes des difficultés de l'enseignement de la géologie (Turki, 2012).

$\mathrm{Au}$ Maroc, les travaux de recherche en didactique qui se sont intéressés à l'enseignement de la géologie sont très peu abordés et sont minoritaires par rapport à ceux qui concernent l'enseignement d'autres disciplines.

Cette synthèse des travaux antérieurs, constitue un modeste jalon marquant le cheminement vers les recherches actuelles. Ainsi notre approche nous paraît essentielle pour compléter une littérature marocaine rarissime et éparse sur les difficultés de l'enseignement et de l'apprentissage de la géologie en classes marocaines secondaires qualifiantes.

\section{Aperçu sur de la disparité de volume horaire entre l'enseignement de la biologie et de la géologie au cycle de l'enseignement secondaire qualifiant.}

A travers une analyse de la disparité de volume horaire entre l'enseignement de la biologie et de la géologie au cycle de l'enseignement secondaire qualifiant au Maroc tel qu'il est décrit dans le document officiel 
relatif aux orientations pédagogiques des sciences de la vie et de la terre (version Juillet 2007), on note que les séances de cours de la géologie sont moins nombreuses que celles de la biologie. Ainsi la $1^{\text {ère }}$ année du baccalauréat sciences expérimentales comporte une seule unité de géologie d'un volume horaire de 34 heures (Tableau 1), programmée au début du $1^{\text {er }}$ semestre contre 3 unités de biologie d'un volume horaire de 102 heures. En $2^{\text {eme }}$ année du Baccalauréat option SVT, la géologie est représentée également par une seule unité de géologie (Tableau 1), programmée vers la fin de l'année scolaire avec un volume horaire de 34 heures contre cinq unités de Biologie d'un volume horaire de 170 heures.

Tandis ce que pour le tronc commun, l'unité «Eau source de vie » comporte quatre chapitres (17 heures) consacrés à l'eau et dont seulement 3heures portent sur l'hydrologie et l'hydrogéologie.

\begin{tabular}{|c|c|c|c|}
\hline Niveau scolaire & $\begin{array}{l}\text { Unités de } \\
\text { géologie }\end{array}$ & $\begin{array}{l}\text { Volume } \\
\text { horaire }\end{array}$ & Objectifs visés (Compétences) \\
\hline $\begin{array}{l}\text { Tronc commun } \\
\text { lettre et science } \\
\text { humaine (TC) }\end{array}$ & $\begin{array}{c}\text { L'eau Source de } \\
\text { Vie }\end{array}$ & $17 \mathrm{H}$ & $\begin{array}{l}\text {-Reconnaitre les sources et l'importance des } \\
\text { ressources en eau. } \\
\text { - Etre conscient de la nécessité de la } \\
\text { rationalisation de l'utilisation des ressources } \\
\text { en eau. }\end{array}$ \\
\hline $\begin{array}{l}\text { Première année } \\
\text { du baccalauréat } \\
\text { Sciences } \\
\text { expérimentales } \\
1 \text { êre année du } \\
\text { baccalauréat } \\
\text { Sciences } \\
\text { mathématiques }\end{array}$ & $\begin{array}{l}\text { Géodynamique } \\
\text { externe }\end{array}$ & $34 \mathrm{H}$ & $\begin{array}{l}\text {-Approfondissement des connaissances en } \\
\text { relation avec les phénomènes de la } \\
\text { géodynamique externe. } \\
\text {-Maitrise des subdivisions du temps } \\
\text { géologique } \\
\text {-Réalisation d'une Lecture systématique de la } \\
\text { cartographie géologique } \\
\text {-Savoir faire appel à des outils appropriés } \\
\text { pour la reconstitution paléogéographique }\end{array}$ \\
\hline $\begin{array}{c}2^{\text {eme }} \text { année du } \\
\text { baccalauréat } \\
\text { Sciences de la } \\
\text { Vie et de la } \\
\text { terre (SVT) } \\
2^{\text {eme }} \text { du } \\
\text { baccalauréat } \\
\text { Sciences } \\
\text { physique } \\
\text { Chimie }\end{array}$ & $\begin{array}{l}\text { Géodynamique } \\
\text { interne }\end{array}$ & $34 \mathrm{H}$ & $\begin{array}{l}\text {-Acquérir des connaissances sur les } \\
\text { phénomènes géologiques associés à la } \\
\text { formation des chaines de montagne. } \\
\text {-Pouvoir se situer dans le temps et dans } \\
\text { l'espace } \\
\text { - Pouvoir lier la tectonique des plaques à la } \\
\text { formation des montagnes. }\end{array}$ \\
\hline
\end{tabular}

Tableau 1. Les unités de la géologie en classes secondaires marocaines

Grâce à cette lecture simplifiée en terme de volume horaire, il apparait clairement que le cursus scolaire secondaire marocain en SVT favorise la biologie et marginalise relativement la géologie en minimisant 
son volume horaire ce qui pourrait être parmi les raisons qui accentuent les difficultés de l'acquisition des concepts géologiques.

Dans le cadre de ce document, nous allons essayer de repérer et investiguer de manière empirique majeure, les difficultés et obstacles se rapportant à l'enseignement de cette discipline au Maroc et qui peuvent se retrouver non seulement chez les élèves mais aussi chez leurs enseignants.

\section{Méthodologie de L'étude}

\section{Présentation de l'échantillon}

Pour tenter de répondre à la problématique citée, nous avons mené cette étude auprès d'un échantillon d'élèves et d'enseignants dans sept établissements scolaires de l'enseignement secondaire qualifiant appartenant à la délégation d'Inzegane Ait Melloul, Académie de Souss Massa et où nos professeurs stagiaires des sciences de la vie et de la terre effectuent leurs stages de Mise en Situation Professionnelles (MSP) d'où leur choix.

L'échantillon d'enseignants comprend 32 enseignants marocains de l'enseignement secondaire qualifiant de la matière des Sciences de la Vie et de la Terre (SVT) ayant accepté de participer à cette enquête. 93,1\% de ces professeurs sont des professeurs de l'enseignement secondaire qualifiant avec une expérience allant de 10 à 30 ans, le reste de l'échantillon est représenté par le corps des professeurs agrégés ayant la même période d'expérience.

L'échantillon des enseignants choisi fait partie d'une population qui correspond à l'ensemble des enseignants des sciences de la vie et de la terre de la délagation Inzegane Ait Melloul. Il est valide car il existe une relation entre l'échantillon et la population et qui réside dans le fait que les enseignants constituant l'échantillon pratiquent dans les mêmes conditions que la population des enseignants.

Quant à l'échantillon d'élèves, il est constitué de 195 élèves du cycle qualifiant avec 41 élèves du Tronc commun, 85 élèves de la première année du baccalauréat et 69 élèves de la deuxième année du baccalauréat. Nous nous sommes focalisés sur les deux années du baccalauréat du fait que leur programme d'SVT consacre une partie à la géologie.

L'échantillon des élèves choisi fait partie d'une population qui correspond à l'ensemble des éleves des classes secondaires qualifiantes de la délgation Inzegane Ait Meloul. Il est valide car il existe une relation entre l'échantillon et la population et qui réside dans le fait que les élèves qui constituent l'échantillon subissant la même formation avec les mêmes moyens didactiques et dans les mêmes conditions que la population des élèves. 


\section{Présentation des Questionnaires D’enquête}

Les questionnaires, format papier, ont été réalisés à partir de l'analyse a priori des thèmes enseignés et des typologies d'obstacles évoquées à travers la synthèse bibliographique des études des difficultés de l'enseignement et de l'apprentissage de la géologie qui ont été menées un peu partout dans le monde, ce qui nous a permis de lister les notions susceptibles de poser les difficultés pressenties en classe secondaire qualifiantes marocaines. Plusieurs questions tant ouvertes que fermées, ont été proposées aux élèves et aux enseignants.

\section{L'évaluation Qualitative de L'outil (Le Questionnaire)}

Après rédaction, et afin de verifier sa validité, le questionnaire est relu par des experts du domaine ; 3 inspecteurs de la matière des SVT, 8 formateurs des futurs enseignants de la matière des SVT au centre régional des métiers de l'education et de la formation Souss Massa et 10 enseignants de la matière SVT pratiquant dans l'enseignement secondaire qualifiant public et/ou privé dans la région de Souss Massa. On les a demandé d'exprimer leurs opinions en termes de langue, de clarté et de planification pour les deux questionnaires et d'apporter leurs appréciations subjectives sur la présence ou absence de dérive par rapport à l'objectif initial, la pertinence des questions (non ambiguïté, planification et ordre, utilité...) et sur la pertinence des modalités de réponse (choix, utilité, nombre...).

À la lumière des commentaires et suggestions des lecteurs, les deux questionnaires ont été rectifiés. On a estimé que ces mesures sont suffisantes pour la fiabilité des deux questionnaires utilisés.

\section{Questionnaire Destiné Aux Professeurs}

Le questionnaire, proposé aux enseignants est précédé d'un recueil de renseignements personnels permettant de définir le profil du professeur concerné, il possède alors 23 questions réparties sur sept principales parties (Tableau 2).

\begin{tabular}{|c|c|c|}
\hline & & Objectifs \\
\hline 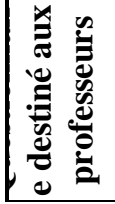 & $\begin{array}{c}\text { Partie 1 } \\
4 \text { Questions }\end{array}$ & $\begin{array}{c}\text { Informations générales sur les profs enquêtés: Cadre (prof de } \\
\text { l'enseignement secondaire qualifiant, prof agrégé) /Spécialité de } \\
\text { licence (Biologie, Biologie-Géologie, Géologie, SVT)/Durée de } \\
\text { travail autant qu'enseignant des SVT/ Qualification (Master, } \\
\text { Doctorat...) }\end{array}$ \\
\hline
\end{tabular}




\begin{tabular}{|c|c|}
\hline $\begin{array}{c}\text { Partie 2 } \\
5 \text { Questions }\end{array}$ & $\begin{array}{c}\text { Formation et Formation continue : Formation professionnelle } \\
\text { en domaine d'enseignement d'SVT (Titulaire du diplôme de } \\
\text { qualification des centres de Formation des enseignants d'SVT, } \\
\text { Insertion directe dans le domaine de l'enseignement) } \\
\text { Formation continue en Géologie : Présente ou non présente/ } \\
\text { Fréquence/ suffisante ou insuffisante } \\
\text { Autoformation en géologie : Présente ou non présente/Outils } \\
\text { d'autoformation }\end{array}$ \\
\hline $\begin{array}{c}\text { Partie } 3 \\
5 \text { Questions }\end{array}$ & $\begin{array}{l}\text { Recommandations officielles et Manuels scolaires d'SVT: Les } \\
\text { instructions traitant l'approche recommandée pour enseigner les } \\
\text { modules géologiques en classe secondaires qualifiantes } \\
\text { /Répartition des unités de géologie/ La séquence des cours de } \\
\text { géologie/ Relation contenu des apprentissages et volume horaire } \\
\text { indiqué dans les curricula officiels /Contenus des cours de } \\
\text { géologie }\end{array}$ \\
\hline $\begin{array}{c}\text { Partie } 4 \\
2 \text { Questions }\end{array}$ & $\begin{array}{c}\text { Ressources pédagogiques utilisés : Type de ressources } \\
\text { pédagogiques mobilisés lors des cours de géologie / Causes et } \\
\text { motifs de non-mobilisation des ressources pédagogiques en cours } \\
\text { de géologie }\end{array}$ \\
\hline $\begin{array}{c}\text { Partie 5 } \\
3 \text { Questions }\end{array}$ & $\begin{array}{c}\text { Sortie de terrain, Expérimentation et modélisation } \\
\text { scientifique : Organisation des sorties pédagogiques et } \\
\text { géologique/ manipuler en classe pour mieux comprendrel } \\
\text { modéliser pour expliquer les phénomènes géologiques } \\
\text { complexes. }\end{array}$ \\
\hline $\begin{array}{c}\text { Partie 6 } \\
3 \text { Questions }\end{array}$ & $\begin{array}{l}\text { L'apport des élèves dans la qualité des apprentissages en } \\
\text { géologie : Motivation et implication /Sensibilités/difficultés } \\
\text { d'apprentissage }\end{array}$ \\
\hline $\begin{array}{c}\text { Partie } 7 \\
1 \text { Question }\end{array}$ & $\begin{array}{l}\text { Solutions proposées pour remédier aux difficultés de } \\
\text { l'enseignement/apprentissage en géologie }\end{array}$ \\
\hline
\end{tabular}

Tableau 2. Présentation du questionnaire destiné aux professeurs

\section{Questionnaire administré aux élèves}

Le questionnaire proposé aux élèves contient 10 questions réparties sur cinq parties (Tableau 3).

\begin{tabular}{|c|c|c|}
\hline & Objectifs \\
\hline \multirow{3}{*}{ 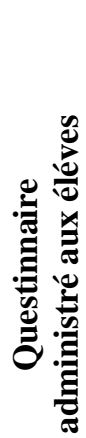 } & $\begin{array}{c}\text { Partie 1 } \\
\text { 3Questions }\end{array}$ & Informations générales sur les élèves: Sexe/ âge/ niveau scolaire \\
\hline & $\begin{array}{c}\text { Partie 2 } \\
3 \text { Questions }\end{array}$ & $\begin{array}{c}\text { ce que représente la géologie pour les élèves : Motivation/Intérêt/ } \\
\text { désintérêt / Moyenne des épreuves de géologie par rapport à celles } \\
\text { de Biologie }\end{array}$ \\
\hline & $\begin{array}{c}\text { Partie 3 } \\
2 \text { Questions }\end{array}$ & $\begin{array}{c}\text { Les élèves face au programme de géologie à enseigner en classe } \\
\text { secondaires qualifiantes: Présence de Difficultés de } \\
\text { l'apprentissage de géologie / les unités de géologie qui présente le } \\
\text { plus de difficultés }\end{array}$ \\
\hline
\end{tabular}




\begin{tabular}{|c|c|c|} 
& $\begin{array}{c}\text { Partie 4 } \\
1 \text { Questions }\end{array}$ & $\begin{array}{c}\text { Identification d'Obstacles sources de difficultés de } \\
\text { l'apprentissage en géologie }\end{array}$ \\
\hline & $\begin{array}{c}\text { Partie 5 } \\
\text { 1Question }\end{array}$ & $\begin{array}{c}\text { Solutions proposées pour remédier aux difficultés de } \\
\text { l'enseignement/apprentissage en géologie }\end{array}$ \\
\hline
\end{tabular}

Tableau 3. Présentation du questionnaire administré aux élèves

\section{Traitements des Résultats de Données D'enquêtes}

Les deux questionnaires étaient anonymes afin d'optimiser le nombre de retours. Après la distribution des questionnaires, 211 ont été complétés par les enseignants et leurs élèves et nous ont été retournés sur les 227 expédiés.

Les réponses des enseignants et des élèves ont été saisies et traitées à l'aide du logiciel IBM. SPSS (Statistical Package for the Social Sciences) Statistic 22. Les pourcentages qui apparaissent dans les différents tableaux joints sont exprimés, en fonction du nombre de personnes interrogées.

\section{Résultats et Discussions}

\section{Questionnaire Dirigé Aux Professeurs}

\section{La Formation et la Formation Continue des Enseignants}

Les enseignants répondant sont nombreux à posséder une formation universitaire complétée par une formation professionnelle au sein des centres de formation des enseignants. 51,7\% d'entre eux sont titulaires d'une licence. 24,1\% sont titulaires d'un diplôme des Ecoles Normales Supérieures (ENS), 10,2\% sont titulaires d'un Diplôme des études supérieures approfondies (DESA), Diplôme des études supérieures spécialisées (DESS) ou Master et 10,7 \% d'un doctorat. Il est également important de relever que seulement $13,3 \%$ des enseignants répondant précisent avoir choisi une voie à dominante sciences de la Terre contre $55,2 \%$ en biologie ou sciences naturelles. Quant à la formation continue, $89,7 \%$ des enseignants qui ont répondu à notre enquête disent avoir bénéficiés des formations continues en relation avec l'enseignement des SVT contre $75,9 \%$ en ce qui concerne l'enseignement de la géologie. Cependant $58,6 \%$ de ces enseignants expriment un manque portant plus particulièrement sur l'enseignement de la géologie et souhaitent la mise à disposition de ressources pour leur formation.

On peut donc regretter que les moyens de formation continue en géologie mis à la disposition des enseignants soient d'une certaine manière déqualifiés et ne concernent qu'une minorité d'entre eux.

Lorsque les enseignants s'expriment sur leur autoformation en géologie, ils disent avoir pour cela utilisés des ressources très diversifiées (conférences, cours et ouvrages universitaires sites internet, revues, expositions scientifiques...). Cependant, les cours et ouvrages universitaires 
ainsi que les sites internet restent une source d'information privilégiée pour la majorité d'entre eux $(75,8 \%)$.

\section{Les Recommandations Officielles et Les Manuels Scolaires D'SVT}

En répondant aux questions se rapportant aux recommandations officielles et aux manuels scolaires d'SVT, on note que $75 \%$ des enseignants soulignent l'absence des instructions qui traitent l'approche recommandée pour enseigner les modules géologiques en classe secondaires qualifiantes. $50 \%$ des enseignants notent que le contenu géologique des manuels scolaires est insuffisant, très abstrait et complexe pour les élèves. De même, $79 \%$ notent une incompatibilité entre les cours programmés et le volume horaire indiqué dans les curricula officiels.

L'insuffisance du contenu géologique et de son volume horaire, déjà évoqué plus haut (cf. tableau $1:$ p 7), ainsi que sa complexité pourraient être parmi les difficultés que rencontre l'enseignement de la géologie au secondaire qualifiant. Ce qui permet de souligner que l'enseignement de cette discipline au Maroc nécessite une réforme structurelle urgente pour relever les défis liés à la qualité de l'enseignement-apprentissage de la géologie en particulier et à la qualité de l'enseignement en général soulignée par la vision stratégique de la réforme (2015-2030) mise en place pour la réforme de l'éducation au Maroc. Le premier élément à prendre en considération dans la démarche de cette réforme structurelle est l'adoption et l'élaboration d'un manuel spécifique à la géologie pour les classes secondaires qualifiantes tout en choisissant des programmes, des thèmes et des unitées géologiques adaptés à ce cycle de l'enseignement tout en faisant appel à la richesse géologique de notre pays le Maroc et en y consacrant un volume horaire suffisant.

\section{Les Ressources Pédagogiques Utilisées Par Les Professeurs}

Un état des lieux des ressources pédagogiques mobilisées lors des pratiques de classe montre que les ressources utilisées par la majorité des enseignants enquêtés (90\%) sont variées. Ainsi ils font appel aux manuels scolaires, aux vidéos et animations (Data Show), aux maquettes, aux cartes (topographiques et géologiques) et aux échantillons géologiques (roches, fossiles, minéraux).

Les causes et les motifs des non-usages des ressources pédagogiques en cours de géologie par certains enseignants (10\%) les relient au manque du temps (emplois de temps chargés, le risque ne pas terminer les programmes...), à la déqualification des outils qui sont mis à leur disposition ou à leur absence totale des établissements scolaires ainsi qu'à l'absence de la maîtrise de l'usage de certains outils par certains professeurs (Matériel géologique, outils technologiques...). 
Ces résultats montrent que les ressources pédagogiques utilisées par la majorité des enseignants sont variées, mais reste à donner plus d'importance au soutien pédagogique et technique des établissements scolaires, et à la mise en disponibilité des ressources nécessaires pour la qualification des enseignants ayant des difficultés de l'usage de certains outils pédagogiques et technologiques.

\section{La sortie de terrain, l'expérimentation et la modélisation scientifique La sortie de terrain}

Selon Orange (2003), la géologie est une science qui s'intéresse d'une part à l'explication de la formation de la terre et d'autre part à son histoire. D'un point de vue méthodologique, ces deux approches renvoient à des démarches différentes. La première privilégie l'investigation au laboratoire et la construction de modèles et la deuxième met plutôt l'accent sur le rôle de l'observation, et en particulier l'observation de terrain.

Les objets et phénomènes géologiques partagent largement un caractère d'inaccessibilité (Allain, 1995). Etant donnée cette inaccessibilité, les enseignants doivent avoir recours, chaque fois qu'ils le peuvent, à des manipulations et à des sorties de terrain. Or dans notre cas d'étude, on constate que $86,2 \%$ des enseignants interrogés ne font pas de sorties de terrain même si la nature des cours enseignés en géologie exigent l'organisation des classes de terrain. Les enseignants relient ce manque des pratiques de terrain à des problèmes liés à la surcharge des classes enseignés, aux moyens de transport, au problème de temps, à l'inaccessibilité parfois des terrains et sites géologiques et au manque des connaissances géologiques de plusieurs enseignants qui ne leurs permettent pas le travail de l'investigation scientifique sur le terrain au cour des sortie géologiques qu'ils organisent. Cette observation va dans le sens des travaux de Sanchez et al. (2005) qui ont montré que les enseignants qui organisent une classe de terrain ont des difficultés à organiser un travail d'investigation scientifique sur le terrain. Dans cette perspective, organiser des sorties géologiques en présence des accompagnateurs et animateurs géologues spécialisés est nécessaire.

Les sorties géologiques de terrain donnent du sens aux apprentissages en favorisant le contact direct avec l'environnement naturel ou culturel, avec des acteurs dans leur milieu de travail, avec des œuvres originales » (Clair \& Gauthier, 2001). Elles permettent une évolution positive des relations entre professeurs et élèves et qui apprennent à se découvrir mutuellement, ce qui est très bénéfique pour l'ambiance de classe (Dalle \& Scandolera, 2000). Pour notre cas, l'insuffisance ou l'absence des sorties de terrain et des manipulations de classe pourraient bien influencer l'évolution positive des 
relations entre professeurs et élèves, ainsi que les apprentissages des concepts géologiques. (Lacoste, 2001; Turki, 2012).

\section{L'expérimentation de Classe en Géologie}

$44,8 \%$ des enseignants interrogés ne font appel aux manipulations que rarement étant donné l'inaccessibilité des phénomènes géologiques étudiés, la surcharge des classes et l'absence de matériel adéquat. Alors que le reste, $55,2 \%$ des enseignants ne mettent jamais en place une expérimentation pédagogique en classe et se limitent à admettre les résultats des manipulations déjà prêtes au niveau des manuels scolaires et relient ceci à la complexité des démonstrations expérimentales et à la lourdeur des effectifs des élèves ne favorisant pas l'acquisition des apprentissages.

La manipulation s'inscrit dans une démarche scientifique qui doit permettre d'élaborer des connaissances et la motivation des élèves (INRP, Sanchez et al 2004). Les élèves doivent faire, et non regarder faire, c'est à ce moment qu'ils sont réellement actifs et que peuvent être remis en question leurs savoirs initiaux. Le manque de ces manipulations et d'observations concrètes peut être l'origine de plusieurs difficultés en relation avec l'enseignement et l'apprentissage de certains thèmes en géologie. Ces résultats obtenus corroborent ceux des études de Turki (2012).

Par ailleurs, certains thèmes géologiques plus abstraits ne se prêtent pas aussi bien à des manipulations concrètes, où l'élève peut toucher, et s'approprier par le vécu, des phénomènes qui jusque là lui étaient restés étrangers. Cependant les documents audiovisuels et la modélisation s'avèrent essentiels.

\section{La Modélisation Scientifique en Géologie}

Quant à la modélisation scientifique qui consiste à construire et à utiliser un modèle qui est une représentation simplifiée de la réalité pour montrer les aspects importants du système étudié ce qui permet l'explication de plusieurs phénomènes géologiques est très peu utilisée $(6,9 \%)$ par les enseignants durant les séances de cours de géologie. Ils lient cette négligence de la modélisation au manque de temps, à l'effectif pléthorique des élèves.

Le modèle est l'outil intellectuel permettant d'accéder à une explication d'un problème scientifique (Orange, 1997). Il est l'instrument de connaissance permettant aux élèves, dans le cadre de la résolution d'un problème scientifique, de conduire leurs investigations et de donner du sens aux tâches dans lesquelles ils s'engagent (Sanchez, 2008). En effet, la perception par les élèves et les enseignants des modèles scientifiques en géologie pourrait simplifier quelques concepts et permettrait d'accéder à une explication des problèmes scientifiques et phénomènes géologiques complexes. Or, le statut du modèle scientifique pose problème aux 
enseignants de sciences de la vie et de la Terre de l'enseignement secondaire qualifiant comme il a été montré dans les travaux de Sanchez et al. (2004 $a b)$.

\section{Les Difficultés de L'enseignement de La Géologie Identifiées Par Les Enseignants}

Les enseignants ayant répondu à notre enquête ont ressenti plusieurs difficultés influençant la qualité de l'enseignement de la géologie en classes secondaires, ainsi $51,7 \%$ des enseignants généralement d'une formation initiale en biologie n'aiment pas enseigner les cours de géologie et relient ceci au fait du manque portant plus particulièrement sur leurs connaissances en géologie et en absence des formations continues.

$93,1 \%$ des enseignants notent que le contenu des cours géologiques à enseigner et des manuels scolaires est impertinent, complexe et très abstrait ce qui pourrait être la cause de l'ennui des élèves et leur désintérêt ce qui en résulte la détérioration de la qualité des apprentissages lors d'une séance de géologie. 86,2\% notent que les apprenants ont des problèmes de se situer dans le temps et dans l'espace. D'autres enseignants relient les difficultés ressentis en enseignant les cours de la géologie aux faibles prérequis des élèves en géologie d'où leur faible niveau $(82,8 \%)$, à l'effectif pléthorique des élèves ne favorisant pas les manipulations et travaux pratiques en classe (43,58\%) ainsi qu'à l'absence des établissements de quelques ressources pédagogiques que souhaitent mobiliser durant ces cours et au faible volume horaire programmé pour les cours de géologie par rapport au cours de biologie $(85,6 \%)$.

D'après ces résultats, il apparait que les obstacles peuvent se retrouver non seulement chez les élèves mais aussi chez leurs enseignants. Ainsi et comme nous l'avons vu plus haut, les majeures difficultés soulignées par les enseignants sont en relation directe avec leurs formations initiales en biologie et manque ou insuffisance des formations continues en géologie. De ce fait, les enseignants peuvent véhiculer des difficultés, d'une manière inconsciente, au cours de l'apprentissage du fait qu'ils possèdent leurs propres conceptions qu'ils risquent éventuellement de transmettre aux élèves (Chalak \& El Hage, 2011). A ceci s'ajoute le contenu abstrait et complexe des cours de géologie, l'insuffisance d'équipements et ressources pédagogique scientifiques et techniques, la lourdeur du programme et volume horaire très faible consacré au cours de la géologie. Ces résultats corroborent ceux obtenus et que nous avons vu plus haut. 


\section{Questionnaire administré aux élèves}

\section{Que représente la géologie pour les élèves ?}

La majorité des élèves qui ont répondu à notre enquête trouvent que leurs notes en géologie sont moyennes par rapport à celles de biologie (tronc commun $82 \%, 1^{\text {ere }}$ année du bac $57,6 \%$ et $58 \%$ du $2^{\text {eme }}$ année du bac).

Les cours de géologie occupent une grande partie du programme de la $1^{\text {ere }}$ année de baccalauréat, néanmoins $65,9 \%$ des élèves de cette année ont des difficultés de compréhension des cours de géologie et notent leur désintérêt vis-à-vis de ces cours de géologie. Toutefois, 56,5\% des élèves de la $2^{\text {eme }}$ année de baccalauréat montrent un bon intérêt pour les cours de géologie et affirment qu'ils n'ont pas de problème de compréhension. L'intérêt porté par ces élèves aux cours géologiques pourrait s'expliquer par l'utilité de ces cours programmés en $2^{\text {eme }}$ année baccalauréat et qui font que les élèves travaillent bien pour réussir leur examen national et avoir leur diplôme.

Ces résultats montrent que les élèves de la $1^{\text {ere }}$ année du baccalauréat sont très peu impliqués dans la géologie ce qui nécessite un encouragement et une motivation.

\section{Les élèves face au programme de géologie à enseigner en classe secondaires qualifiantes}

Les cours de géologie que les enseignants ont à enseigner en classes secondaires qualifiantes sont variés. Ainsi le programme du tronc commun lettre laisse les élèves à découvrir quelque notions sur les ressources en eaux, un chapitre de la géodynamique externe est consacré pour la première année du baccalauréat alors que le programme de la géologie de la $2^{\text {eme }}$ année porte sur la géodynamique interne dont certaines notions ont été reconduites en faisant appel aux connaissances des élèves.

Afin de souligner les possibles cours montrant des difficultés de compréhension en relation avec les obstacles cité en bibliographie et qui sont liés au temps, à l'espace et au modèle, les élèves ayant répondu et en fonction de leur niveau d'étude indiquent en effet avoir ressenti quelques difficultés. Ainsi 47,3\% des élèves du tronc commun attestent avoir rencontrés des difficultés de compréhension des concepts qui sont en relation avec les ressources en eau et eaux souterraines en reliant ces difficultés d'apprentissage à l'absence du model scientifique pouvant faciliter les phénomènes géologique. $48,2 \%$ des élèves de la 1ere année du bac témoignent des difficultés en ce qui concerne les cours de la sédimentation et des milieux sédimentaires, $40 \%$ pour les cours de stratigraphie (stratotype, lacune stratigraphique) et $32,9 \%$ des cours de la reconstitution des paléo environnements. Toutefois $71,8 \%$ des élèves attestent des difficultés de compréhension de la coupe géologique. Tandis ce 
que plus de $80 \%$ des élèves de la $2^{\text {eme }}$ année du bac considèrent que les cours proposés ne leur posent aucun problème de compréhension.

Ces résultats montrent que les élèves éprouvent des difficultés pour la compréhension des cours de la géodynamique externe programmés lors de la $1^{\text {ère }}$ année du baccalauréat, et ce, plus particulièrement pour la sédimentologie (sédimentation et milieux sédimentaires), la stratigraphie (Datation et reconstitution des paléo environnements) et de la cartographie (carte géologique et coupe géologique). On peut ainsi expliquer ces problèmes de compréhension posés par des difficultés qu'ont les élèves à appréhender les temps longs et la vitesse de réalisation des phénomènes géologiques comme l'ont montré plusieurs travaux antérieurs (Gould, 1990; Gohau, 1990; Trend, 2000; Sanchez \& Prieur, 2006; Raab \& Frodeman, 2002), au fixisme qui constitue un obstacle majeur à la compréhension de phénomènes dynamiques paraissant statiques à l'échelle humaine (Monchamp et al., 1995), à l'insuffisance des ressources pédagogiques mobilisées, l'absence de sorties géologiques ainsi qu'au manque des prérequis nécessaires chez les élèves des classes secondaires qualifiantes comme on l'a déjà évoqué plus haut.

\section{Les difficultés de la compréhension de géologie identifiées par les élèves}

Plus de $60 \%$ des élèves relient leurs difficultés de compréhension de la géologie aux méthodes inefficaces d'enseignement adaptées en classe par leurs professeurs, à l'absence, défaillance ou non mobilisation des ressources pédagogiques et la non intégration des TICE. 81,5\% relient leurs difficultés d'apprentissage à l'absence de sorties de terrains et des travaux pratiques en relation avec les cours de la géologie ou à la surcharge des classes.

Ainsi les difficultés des apprentissages de la géologie sont d'après les élèves liés à la surcharge des classes, à l'absence des sorties et manipulation de classe ainsi qu'à la négligence de la modélisation scientifique et la non intégration des TICE dans les pratiques enseignantes en géologie. Ces résultats confirment les conclusions déjà évoqué plus haut.

\section{Solutions Proposées}

Face aux diverses difficultés rencontrées lors de l'enseignement et l'apprentissage de la géologie en classe secondaires qualifiantes, et dans le but de les surmonter, les principales solutions proposées par les élèves et leurs enseignants convergent vers les mêmes points à savoir une transposition didactique convenable et une simplification du contenu et concepts géologiques par la mobilisation des ressources pédagogiques tels que les modèles géologiques, l'augmentation du volume horaire alloué à l'enseignement de la géologie dans l'enseignement secondaire, l'adaptation de la manipulation de classe, les travaux pratiques et les sorties de terrain 
ainsi que l'intégration des TICE en mobilisant des ressources numériques qui contribuent à renouveler les modes de représentation de l'espace avec des illustrations en $2 \mathrm{D}$ et $3 \mathrm{D}$ et en effectuant des rotations afin d'accéder à une explication des problèmes scientifiques et phénomènes géologiques complexes, ou encore mettre en place des sessions de la formation continue pour les enseignants permettant la mise à niveau de leurs connaissances en géologie et pouvant les aider à améliorer les méthodes et techniques de l'enseignement ainsi que les méthodes d'acquisition des connaissances chez les élèves dans l'enseignement de cette science (Gilles Aldon et al., 2010) (Figure 1).

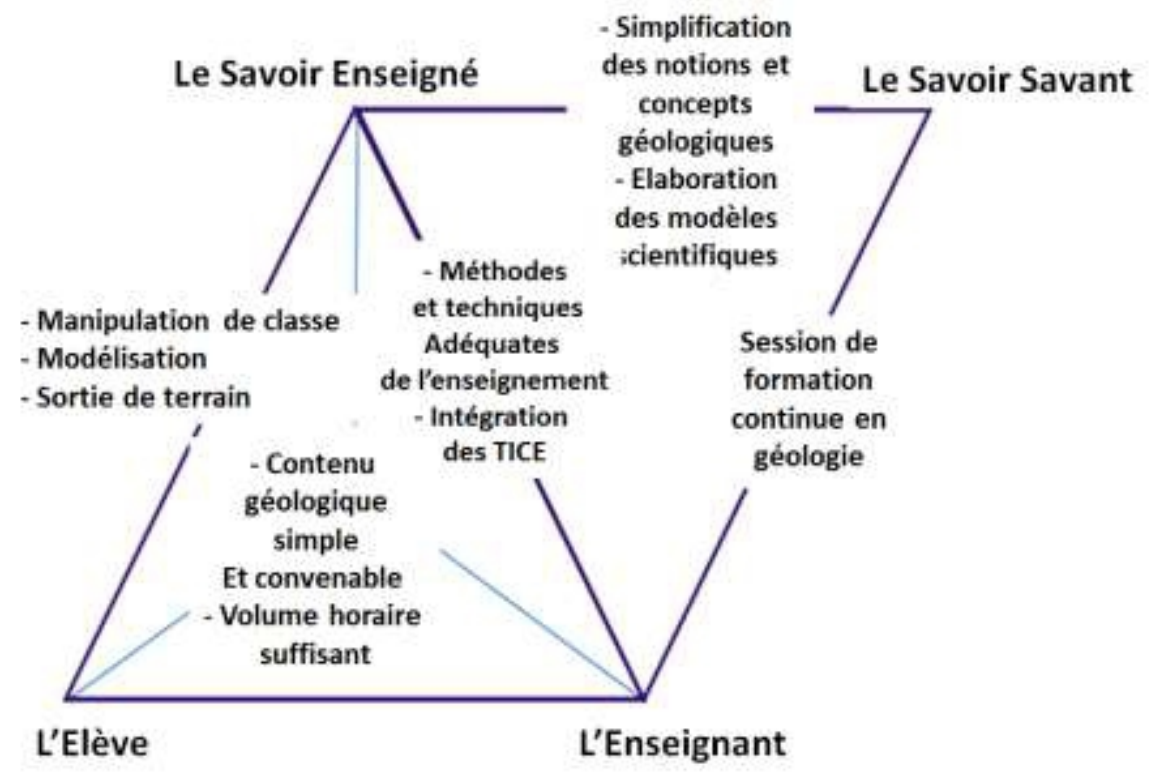

Figure 1. Solutions proposées

\section{Conclusion}

Les résultats de la présente étude montrent que l'enseignement et la compréhension de la géologie en classes secondaires qualifiantes marocaines, cas de la délégation Inzegane Ait Melloul, sont confrontés à plusieurs difficultés aussi bien pour les élèves que pour les enseignants. Ainsi les moyens de la formation continue en géologie qui sont mis à la disposition des enseignants sont déqualifiés et ne concernent qu'une minorité d'entre eux, en plus de l'insuffisance du contenu géologique, de son volume horaire et sa complexité, de l'absence ou la non mobilisation des ressources pédagogiques et du faible prérequis des élèves en géologie. Ces difficultés sont largement liées aux rapports que la discipline entretient avec l'espace et le temps long, et qui font que les élèves trouvent des difficultés à appréhender plusieurs phénomènes géologiques complexes relatifs à la 
stratigraphie, la paléogéographie et à la conception de la coupe géologique. L'insuffisance ou l'absence des sorties de terrain, l'absence des manipulations et de la modélisation scientifique des phénomènes géologiques complexes dans la majorité des classes interrogées, influencent les apprentissages de la géologie ainsi que l'intérêt porté par les élèves vis-àvis de ces cours ce qui la rend, une matière ennuyeuse et mal aimée par la majorité des élèves. Cependant les pratiques de classe nécessitent à donner plus d'importance à l'utilisation des ressources pédagogiques entre autre les moyens audiovisuels et modèles géologiques. Leur perception par les élèves et les enseignants pourrait simplifier quelques concepts et permettrait d'accéder à une explication des problèmes scientifiques et phénomènes géologiques complexes. Les sorties de terrain et les manipulations de classe en parallèle de l'intégration des TICE permettraient une bonne situation dans le temps et dans l'espace aux élèves. Cette situation n'est pas sans problèmes pour les enseignants qui doivent parfois enseigner la géologie sans avoir été eux-mêmes formés à l'enseignement de cette discipline, ce qui nécessite la mise en place des sessions de formation continue pour les enseignants afin d'améliorer leurs connaissances en géologie pour pouvoir développer des méthodes et des techniques efficaces d'acquisition des connaissances chez leurs élèves. Le fait que cette science soit réduite des programmes scolaires marocains nécessite une réflexion sérieuse de la part des concepteurs des programmes des Sciences de la Vie et de la Terre, des spécialistes de la discipline et du ministère de l'Education Nationale tout en réarrangeant les unités de la géologie et en élaborant un programme de géologie propre au Maroc vu sa richesse géologique et en adoptant un manuel spécifique à la géologie pour les classes secondaires qualifiantes en favorisant les activités permettant de mettre en oeuvre une véritable démarche d'investigation et de modélisation, ce qui pourrait valoriser l'enseignement et l'apprentissage de cette matière, clefs de la réussite scolaire.

\section{References:}

1. Aldon, G., Genevois, S., \& Sanchez, E. (2010). Construction de l'espace et visualisation d'images numériques : regards croisés entre différentes disciplines. In M. Coquidé \& M. Prieur (Eds.), Enseigner l'espace et temps à l'école et au collège : obstacles, pratiques, outils (pp. 141-171). Lyon: INRP.)

2. A. Turki Bin Abdullah (2012). La géologie dans les programmes du ministère de l'éducation et de la formation. Association Saoudienne des Sciences de la Terre 
3. Chalak Hanaà \& Fadi El Hage (2011). L'enseignement des sciences de la Terre au Liban: enjeux, obstacles et orientations professionnelles, RDST, 3 | 2011, 209-240

4. Claire, S. \& Gauthier, D (2001). Les sorties éducatives IUFM de l'Académie de Montpellier.

5. Dalle, H \& Scandolera, M (2000). Sorties scolaires : un outil pour les apprentissages, mémoire professionnel. IUFM de Montpellier.

6. Dictionnaire LAROUSE, Définition de la géologie

7. Gould, S-J. (1990). Aux racines du temps. Grasset et Fasquelle.

8. Gohau G. (1987/1990). Une histoire de la géologie. Paris : Seuil.

9. Lacoste C. (2001). Influence des travaux de terrain sur les apprentissages en géologie, 382 pages, didactique de la géologie, thèse de doctorat de l'Université de Limoges.

10. Lakhloufi A. (2014). Pourquoi l'étude de la géologie n'as pas réussi au Maroc? La semaine de la presse (Alousboua assahafi) 26 Mars. Centre des Etudes et des Recherches de l'Evaluation, de l'Education et de la Formation

11. Disponible ne ligne à : http://www.alousboue.com/4180/4180/

12. Lamarti L., A. ben-bouziane, H. akrim \& M. talbi (2009) : La sortie de terrain : quelle place et quel rôle dans une démarche scientifique ?. RADISMA, Numéro 4 (2009). 15 décembre 2009, http://www.radisma.info/document.php?id=702. ISSN 1990-3219

13. Ministère de l'Education Nationale et de la Formation Professionnelle, Direction des curricula. Programmes et orientations pédagogiques générales relatives au cycle de l'enseignement secondaire qualifiant au Maroc. Juillet (2007), Pages 16-23-27-3339

14. Monchamp A. \& Sauvageot- Skibine M. (1995). Du fixisme à la tectonique des plaques. Et pourtant elles bougent. Aster, $\mathrm{n}^{\circ} 20$, p. 420.

15. Orange C., Beorchia F., Ducrocq P. \& Orange D. (1999). "Réel de terrain », "Réel de laboratoire » et construction des problèmes en sciences de la vie et de la Terre. Aster, $n^{\circ} 28$, p. 107-129.

16. Orange D. (2003). Utilisation du temps et explications en sciences de la Terre par les élèves de lycée : étude dans quelques problèmes géologiques. Thèse de doctorat de l'Université de Nantes.

17. Raab T. \& Frodeman R. (2002). What's it like to be a geologist? Phenomenology of geology and its practical implications. Philosophy and Geography, 5, p. 69-81.

18. Sanchez E. (2008). Quelles relations entre modélisation et investigation scientifique dans l'enseignement des sciences de la terre? », Éducation et didactique, vol 2 - n² | 2008, 93-118. 
19. Sanchez E. \& Prieur M. (2006). Démarche d'investigation dans l'enseignement des sciences de la Terre: activités élèves et scénarios. Scénariser l'enseignement et l'apprentissage: une nouvelle compétence pour le praticien? 8ème Biennale de l'éducation. Lyon : INRP/APRIEF.

20. Sanchez, E \& Fontanieu V (2005). L'enseignement des sciences de la Terre : Que font les élèves sur le terrain? Actes XXVIIèmes JIES. Chamonix.

21. Sanchez E., Prieur M. \& Devallois D. (2004a). Formation initiale et continue des enseignants en sciences de la Terre : Quels besoins pour quelle évolution des pratiques? 7ème Biennale de l'éducation. Lyon

22. Sanchez E., Prieur M. \& Devallois D. (2004b). Fonder la formation sur l'analyse des pratiques, besoins exprimés vs besoins réel : le cas de l'enseignement des sciences de la Terre au lycée. Bordeaux.

23. Sanchez E., Michèle Prieur \& Daniel Devallois (2004). Rapport de recherche L'enseignement des sciences de la Terre en classe de seconde pratiques de classe, difficultés, perspectives pour la formation: Institut National de Recherche Pédagogique

24. Trend R. (2000). Conceptions of geological time among primary teacher trainees, with reference to their engagement with geoscience, history, and science. International Journal of Sciences Education, vol 22.

25. Vision stratégique de la réforme 2015-2030: version français disponible en ligne à http://www.csefrs.ma/pdf/Vision_VF_Fr.pdf 